\title{
RESEARCH
}

Open Access

\section{Breath-hold and free-breathing quantitative assessment of biventricular volume and function using compressed SENSE: a clinical validation in children and young adults}

Murat Kocaoglu' ${ }^{1}$, Amol S. Pednekar ${ }^{1,2^{*}}$ (D) Hui Wang ${ }^{1,2,3}$, Tarek Alsaied ${ }^{4,5}$, Michael D. Taylor ${ }^{4,5}$ and Mantosh S. Rattan ${ }^{1,2}$

\begin{abstract}
Background: Although the breath-hold cine balanced steady state free precession (bSSFP) imaging is well established for assessment of biventricular volumes and function, shorter breath-hold times or no breath-holds are beneficial in children and severely ill or sedated patients.

Methods: Clinical cardiovascular magnetic resonance (CMR) examinations from September 2019 to October 2019 that included breath-hold (BH) and free-breathing (FB) cine bSSFP imaging accelerated using compressed sensitivity encoding (C-SENSE) factor of 3 in addition to the clinical standard BH cine bSSFP imaging using SENSE factor of 2 were analyzed retrospectively. Patients with structurally normal hearts who could perform consistent BHs were included. Aortic flow measured by phase contrast acquisition was used as a reference for the left ventricular (LV) stroke volume. Comparative analysis was performed for evaluation of biventricular volumes and function, imaging times, quantitative image quality, and qualitative image scoring.

\footnotetext{
* Correspondence: Amol.pednekar@cchmc.org

'Department of Radiology, Cincinnati Children's Hospital Medical Center, 3333 Burnet Ave, Cincinnati, OH 45229, USA

2Department of Radiology, University of Cincinnati College of Medicine, Cincinnati, OH, USA

Full list of author information is available at the end of the article
}

(c) The Author(s). 2020 Open Access This article is licensed under a Creative Commons Attribution 4.0 International License, which permits use, sharing, adaptation, distribution and reproduction in any medium or format, as long as you give appropriate credit to the original author(s) and the source, provide a link to the Creative Commons licence, and indicate if changes were made. The images or other third party material in this article are included in the article's Creative Commons licence, unless indicated otherwise in a credit line to the material. If material is not included in the article's Creative Commons licence and your intended use is not permitted by statutory regulation or exceeds the permitted use, you will need to obtain permission directly from the copyright holder. To view a copy of this licence, visit http://creativecommons.org/licenses/by/4.0/ The Creative Commons Public Domain Dedication waiver (http://creativecommons.org/publicdomain/zero/1.0/) applies to the data made available in this article, unless otherwise stated in a credit line to the data. 
(Continued from previous page)

Results: There were 26 patients who underwent all three cine scans during the study period (16.7 \pm 6.4 years, body surface area (BSA) $1.6 \pm 0.4 \mathrm{~m}^{2}$, heart rate $83 \pm 7$ beats/min). BH durations of $8 \pm 1 \mathrm{~s}$ with C-SENSE $=3$ were

significantly shorter $(p<0.001)$ by $33 \%$ compared to $12 \pm 1 \mathrm{~s}$ with SENSE $=2$. Actual scan time for BH SENSE $(4.9 \pm$ $1.2 \mathrm{~min}$ ) was comparable to that with FB C-SENSE (5.2 $\pm 1.5 \mathrm{~min} ; p=\mathrm{NS})$. Biventricular stroke volume and ejection fraction, and LV mass computed using all three sequences were comparable. There was a small but statistically significant $(p<0.05)$ difference in LV end-diastolic volume $(-3.0 \pm 6.8 \mathrm{ml})$ between BH SENSE and FB C-SENSE. There was a small but statistically significant $(p<0.005)$ difference in end-diastolic LV $(-5.0 \pm 7.7 \mathrm{ml})$ and $\mathrm{RV}(-6.0 \pm 8.5 \mathrm{ml})$ volume and end-systolic LV $(-3.2 \pm 4.3 \mathrm{ml})$ and RV $(-4.2 \pm 6.8 \mathrm{ml})$ volumes between BH C-SENSE and FB C-SENSE. The LV stroke volumes from all three sequences had excellent correlations $(r=0.96$, slope $=0.98-1.02)$ with aortic flow, with overestimation by $2.7(5 \%)$ to $4.6(8 \%) \mathrm{ml} /$ beat. The image quality score was Excellent (16 of 26) to Good (10 of 26) with BH SENSE, Excellent (13 of 26) to Good (13 of 26) with BH C-SENSE, and Excellent (3 of 26) to Good (21 of 26) to Adequate (2 of 26) with FB C-SENSE.

Conclusions: Image quality and ventricular volumetric and functional indices using either BH or FB C-SENSE cine bSSFP imaging were comparable to standard BH SENSE cine bSSFP imaging while maintaining nominally identical spatio-temporal resolution. This accelerated image acquisition provides an alternative to accommodate patients with impaired $\mathrm{BH}$ capacity.

Keywords: Compressed SENSE, Left ventricular indices, Right ventricular indices, Free-breathing cine, Pediatric, Children

\section{Introduction}

The assessment of cardiac volumetric indices is important for the diagnosis and follow-up of both congenital and acquired heart disease [1-6]. Cardiovascular magnetic resonance (CMR) imaging is an accurate and reproducible modality that is the clinical reference standard for quantitative evaluation of ventricular chamber size, function and myocardial mass [6-11]. Currently, retrospectively cardiac gated two-dimensional segmented k-space cine balanced steady state free precession (bSSFP) is the preferred CMR sequence for the quantitative assessment of cardiac function. The bSSFP sequence has high intrinsic blood pool to myocardium contrast and high signal-to-noise ratio (SNR) that results in well-defined endocardial boundaries throughout the cardiac cycle [12-14]. Short-axis (Sax) cine bSSFP images are routinely acquired during breath-holds, because the bSSFP sequence is susceptible to artifacts from respiratory motion and disruption of magnetization steady state [14]. In routine clinical practice, one to three cine SAx slices are acquired in a breath-hold (BH) of 5 to15 cardiac cycles by trading the intrinsic high bSSFP signalto-noise (SNR) for imaging speed using parallel imaging techniques that employ regular k-space undersampling in the spatial dimensions e.g. sensitivity encoding (SENSE), without compromising the blood to myocardial contrast and providing adequate spatio-temporal resolution $[15,16]$. Although the accuracy and reproducibility of CMR bSSFP for the measurement of ventricular volumes, function, and cardiac mass is well established [17-19], the requirement for repeated $\mathrm{BHs}$ remains a limitation, especially in children and sedated patients. Accelerated cine CMR techniques (such as k-t BLAST, TPAT, TSENSE, and compressed sensing) have reported bias in left ventricular (LV) volumes, function, and LV mass due to spatiotemporal blurring and temporal filtering [20-26]. Free-breathing (FB) respiratory triggered retrospectively cardiac gated cine bSSFP sequences have been reported to provide biventricular volumes, function, and LV mass comparable to $\mathrm{BH}$ acquisitions with a SENSE acceleration factor of 2 in adults and children [27, 28]. With the goal of accelerating SENSE, a compressed sensitivity encoding (C-SENSE) algorithm was developed that employs a pseudorandom undersampling of $\mathrm{k}$-space in the spatial domain. C-SENSE provides diagnostic CMR image quality with acceleration factors greater than 2 , allowing for significantly reduced $\mathrm{BH}$ times [29]. However, there is little data validating quantitative ventricular assessment using $\mathrm{C}$ SENSE either with BH or FB acquisitions [30].

The purpose of our study was to test the hypothesis that the use of C-SENSE acceleration in $\mathrm{BH}$ and FB respiratorygated retrospectively cardiac gated cine bSSFP sequences produces diagnostic quality images and accurate ventricular volumetric indices with decreased $\mathrm{BH}$ times.

\section{Materials and methods}

This HIPAA-compliant, retrospective study was approved by the institutional review board (IRB) at our institution. The requirement for informed consent was waived. The free-breathing cine bSSFP sequence in its current form was implemented within our institution, and all the data and information were always under the control of our institution.

\section{Patients}

We identified all patients who had undergone clinically indicated CMR examinations that included BH SENSE, 
BH C-SENSE, and FB C-SENSE sequences between September 2019 and October 2019. During this period, CSENSE was used as part of a quality improvement effort to shorten and/or eliminate $\mathrm{BH}$ in CMR acquisition protocols. IRB approval was obtained for the current study which involved systematic retrospective review of those images previously obtained for clinical quality improvement. Patients with congenital heart disease and those who could not complete all three scans were not included.

\section{CMR technique}

All CMR examinations were performed with a $1.5 \mathrm{~T}$ CMR scanner (Ingenia, Philips Healthcare, Best, The Netherlands). SAx cine bSSFP acquisitions covering the entire heart were performed using vector electrocardiogram gating with a dedicated 28 element torso coil and a respiratory bellows placed at the mediastinum, as in routine clinical CMR sessions. All cine imaging was performed prior to administration of contrast agents. $\mathrm{BH}$ cine SAx acquisitions were performed with a SENSE acceleration factor of 2, followed by a second acquisition with a C-SENSE acceleration factor of 3. A third SAx cine acquisition was performed with the cardiorespiratory synchronized $[27,28]$ FB sequence using Fixed mode (one cardiac cycle per respiration) with a CSENSE acceleration factor of 3 . No special breathing instructions were given during the FB acquisition. All three retrospectively cardiac gated SAx cine acquisitions were performed with identical imaging parameters. The imaging parameters were: repetition time (TR) ms/echo time (TE) ms, 2.5-2.7/1.25-1.35; flip angle (FA), $60^{\circ}$; acquired voxel size, $1.6-1.7 \times 1.6-1.7 \times 6-8 \mathrm{~mm}^{3}$ (zero gap); acquired temporal resolution, $40-45 \mathrm{~ms}$. Actual breath-hold durations and acquisition times were extracted from the scanner log files. As part of the standard clinical protocol, quantitative flow assessment (TR/ $\mathrm{TE}-4.5 / 2.7$; FA, $12^{\circ}$; acquired voxel size, $1.6-1.7 \times 1.6-$ $1.7 \times 6 \mathrm{~mm}^{3}$; acquired temporal resolution, $40-45 \mathrm{~ms}$; velocity encoding, $150-200 \mathrm{~cm} / \mathrm{s}$ ) of the aorta at the level of sinotubular junction was performed.

Commercially available implementation of SENSE and CSENSE reconstruction were used. The SENSE algorithm employs data consistency based on a regular undersampling pattern and coil sensitivity information, and spatial solution space constraint based on prior knowledge of the image extent [31]. The C-SENSE combines a spatial domain pseudo-random undersampling pattern of $\mathrm{k}$-space with the SENSE reconstruction algorithm using iterative reconstruction and sparsity constraints [31]. Both these techniques required coil sensitivity and noise estimation information from data acquired during the pre-scan. The 3D pre-scan with coil specific field of view and spatial resolution is performed in a single $7 \mathrm{~s}$ breath-hold, equivalent to single slice acquisition, and information is used for the entire SAx stack. No additional BH is incurred if pre-scan information collected for the localizers and previous scans is compatible with the field of view prescribed for the cine SAx stack and table and patient position is unchanged. The regularization parameters are automatically adjusted for individual patient body habitus, coil topology, and SNR of the prescribed sequence. Specifically, for cine bSSFP sequence, the transient phase for bSSFP is initialized by an $\alpha / 2-\mathrm{TR} / 2$ preparation followed by an alternating radiofrequency phase scheme that generates a steady-state $[14,32]$. The ky undersampling pattern is determined by a pseudo-random variable density Poisson distribution for the prescribed field of view and spatial resolution. Based on the prescribed temporal resolution of a single cardiac phase, this ky pattern is then divided into multiple sequential $k$-space segments of an equal number of ky lines. Thus, the phase encoding gradient amplitude change is minimal close to the center of $\mathrm{k}$-space and signal instabilities due to eddy currents are confined to the periphery of k-space [32]. The k-space segment is repeated for each cardiac phase within a cardiac cycle and subsequently used for retrospective cardiac gating.

\section{Image analysis}

All images were transferred to a separate post-processing workstation (Medis Suite 3.1, Medis Medical Imaging Systems, Leiden, The Netherlands). The SAx cine images were analyzed by a single reviewer (MK), under the supervision of a cardiac radiologist (MR) with $>6$ years of experience who reviewed all measurements. The quantitative assessment of the LV and right ventricular (RV) volumetric indices (end-diastolic volume, end-systolic volume, stroke volume, ejection fraction) and LV mass was performed using the SAx cine bSSFP images. End diastolic and end systolic phases were defined at the midventricular level. For all SAx cine bSSFP series LV basal slices were defined when at least $50 \%$ of the myocardium was visible next to the mitral valve [33, 34]. The RV basal slice was defined below the pulmonary valve and the inflow tract areas were excluded if surrounding myocardial muscle was thin and not trabeculated, suggestive of right atrium [35]. The apical ventricular slices were defined as the last slice showing intracavity blood pool. All endocardial and epicardial contours were drawn manually. The papillary muscles were not contoured and were assigned to the ventricular cavities [36]. Regions of interests were drawn inside the LV blood pool and septal myocardium in diastole to compute the normalized bloodto-myocardial contrast. Image quality was graded independently by three CMR readers (MK, MR and TA with > 3 years' experience in CMR). The image quality scores were based on three criteria: blood-to-myocardial contrast $(\mathrm{BMC})$, endocardial edge delineation (EED), and presence of artifacts such as bulk motion artifacts and residual undersampling related artifacts. Each criterion was graded 
on a scale of 1 to 5 , where 1 was nondiagnostic, 2 was suboptimal but still diagnostic for volumetric analysis, 3 was adequate, 4 was good, and 5 was excellent. All the slices were reviewed individually and an average image quality score for each criterion was assigned to the entire SAx stack. The total combined image quality score was calculated as the average of the three scores. All the patient data were included in the analysis.

\section{Data analysis}

Descriptive statistics of continuous quantitative measurements were summarized as means and standard deviations. Bland-Altman analysis [37] and the two-sided paired $t$ test were used to compare each of the parameters computed using standard of care BH SENSE with those computed using $\mathrm{BH}$ and FB C-SENSE acquisitions. LV stroke volume computed using SAx cine imaging and aortic quantitative flow were compared using Bland-Altman analysis. A $p$-value $<0.05$ was considered significant for all inference testing and $95 \%$ confidence intervals were calculated as appropriate. Tukey multiple comparison analyses and Tukey box plots [38] were used to compare scan time, $\mathrm{BMC}$ normalized to myocardial signal, and image quality scores between three cine bSSFP acquisitions. The Kruskal-Wallis test was performed to compare differences in image quality scores between readers and between scoring criteria. Wilcoxon signed-rank tests were performed to compare image quality scores assigned to the $\mathrm{BH}$ SENSE, BH C-SENSE, and FB C-SENSE acquisitions. For each of the three image quality scoring criteria considered in the study, the percentage of clinical subjects who received a range of image quality scores was plotted as a bar graph. All statistical analyses were performed using MATLAB (The MathWorks ${ }^{\mathrm{TM}}$ Inc., Natick, Massachusetts, USA).

\section{Results}

There were 26 patients (26 males; $16.7 \pm 6.4$ years (range: 9-35), body surface area (BSA) $1.6 \pm 0.4$ (range: 0.94$2.4 \mathrm{~m}^{2}$ ) and heart rate $83 \pm 7.2$ beats/min (range: $50-$ 115)) who underwent all three SAx cine scans during the study period. Table 1 summarizes the patient characteristics. Indications for CMR included Duchenne muscular dystrophy $(n=12)$, pectus excavatum $(n=10)$, Becker muscular dystrophy $(n=1)$, Marfan syndrome $(n=1)$, Turner syndrome $(n=1)$, and chemotherapy induced cardiomyopathy $(n=1)$. All SAx cine images were obtained without technical failure or significant artifact. A total of $15 \pm 2$ (range: $12-18$ ) SAx slices were acquired per patient in $8 \pm 1$ breath-holds ( 2 slices per breathhold) (range: 6-9). BH durations of $8 \pm 1$ (range: 7-10) sec with C-SENSE $=3$ were significantly shorter $(p<$ 0.001 ) by $33 \%$ compared to $12 \pm 1 \mathrm{~s}$ (range: $10-14$ ) with SENSE $=2$. Actual image acquisition time, including $\mathrm{BH}$
Table 1 Characteristics of the study population

\begin{tabular}{ll}
\hline Number of patients & 26 \\
\hline Age (years) & $16.7 \pm 6.4(9-35)$ \\
Female-to-male ratio & $6: 20$ \\
Height (cm) & $154.3 \pm 21.9(121-191)$ \\
Weight (kg) & $61 \pm 26(24-134)$ \\
BSA ( $\left.{ }^{2}\right)^{2}$ & $1.59 \pm 0.38(0.94-2.44)$ \\
Heart rate (beats/min) & $83 \pm 17(50-115)$ \\
Clinical Indications & \\
$\quad$ Duchenne muscular dystrophy & 12 \\
Pectus excavatum & 10 \\
Becker muscular dystrophy & 1 \\
Marfan syndrome & 1 \\
Turner syndrome & 1 \\
Chemotherapy induced cardiomyopathy & 1 \\
\hline
\end{tabular}

The data are presented as the mean \pm standard deviation (minimum maximum) or as the number of subjects

$B S A$ body surface area

instructions and time between BHs, for the SAx stack with $\mathrm{BH}$ SENSE $=2(4.9 \pm 1.2 \mathrm{~min}$ (range: $2.6-7))$ was significantly longer $(p<0.05)$ compared to that with $\mathrm{BH}$ C-SENSE $=3(4.3 \pm 1.5 \mathrm{~min}$ (range: $2.1-7))$ and comparable to that with FB C-SENSE $=3(5.2 \pm 1.5 \mathrm{~min}$ (range: 2.3-7)). Figure 1a depicts the comparison of scan time per slice.

Table 2 demonstrates the data comparing LV and RV volumetric indices measured with the three sequences. $\mathrm{LV}$ and RV stroke volume and ejection fraction, and LV mass computed were comparable. There were no significant differences in RV volumes, and LV and RV stroke volumes, ejection fractions, or myocardial mass between BH SENSE and BH C-SENSE sequences. There was a small but statistically significant difference in $\mathrm{LV}$ and RV ventricular end diastolic and end systolic volumes between BH C-SENSE and FB C-SENSE sequences. Figure 2 depicts linear regression and Bland-Altman plots comparing LV stroke volume computed using aortic quantitative flow with those computed using three cine bSSFP SAx acquisitions. The LV stroke volumes from the three sequences had excellent correlations with aortic flow, regression slopes ranged from 0.98 to 1.02 . All three sequences overestimated the LV stroke volume by $2.7(5 \%)$ to $4.6(8 \%) \mathrm{ml} /$ beat compared with the aortic phase contrast data. The limits of agreement of LV stroke volumes for all three sequences was less than $24 \%$.

BMC normalized to myocardial signal for FB CSENSE was significantly lower $(p<0.05)$ than with both $\mathrm{BH}$ SENSE and BH C-SENSE acquisitions (Fig. 1b). Image quality scores were comparable between the three readers across all criteria in all sequences. The mean of three observers' scores in each criterion were used for 

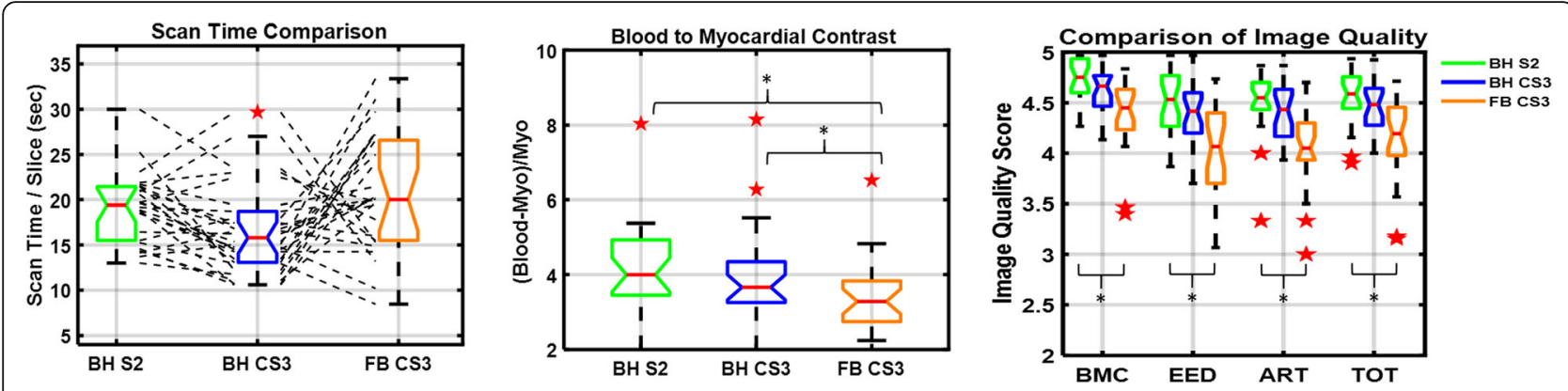

Fig. 1 Tukey box plots of (a) imaging duration, (b) blood-to-myocardial contrast (BMC) normalized to myocardial signal, and (c) image quality scores for three short-axis acquisitions. Imaging duration includes gaps between breath-holds. One-to-one line plots (dotted black lines) for imaging duration with breath hold (BH) C-SENSE $=3$ and free breathing (FB) C-SENSE $=3$ depict the dependence of imaging duration on the patient's heart rate-to-respiratory rate ratio. BMC of FB C-SENSE $=3$ scan was significantly lower $(p<0.05)$ than both BH SENSE $=2$ and BH CSENSE $=3$ acquisitions. Center red line $=$ median, whiskers $=$ minimum and maximum within 1.5 times interquartile distance, red $\star=$ outliers beyond 1.5 times the interquartile distance. Non-overlapping notches indicate that the medians of the two groups differ at the $5 \%$ significance level. Black * with bracket indicates two groups are significantly different $(p<0.05)$. ART $=$ artifacts; CS3 C-SENSE acceleration factor of 3; EED = endocardial edge delineation; S2 SENSE acceleration factor of 2; TOT = total combined score

Table 2 Left and Right Ventricular Volumetric Indices and Difference in their Values between Breath-hold with SENSE Acceleration Factor of 2, Breath-hold with C-SENSE Acceleration Factor of 3, and Free-breathing Sequence with C-SENSE Acceleration Factor of 3 (All Subjects)

\begin{tabular}{|c|c|c|c|c|c|c|c|c|c|}
\hline & \multicolumn{3}{|c|}{ Imaging Sequence } & \multicolumn{3}{|l|}{ Difference } & \multicolumn{3}{|l|}{$\boldsymbol{P}$ value } \\
\hline & BH S2 & $\mathrm{BH}$ CS3 & FB CS3 & $\begin{array}{l}\text { BH S2 - BH } \\
\text { CS3 }\end{array}$ & $\begin{array}{l}\text { BH S2 - FB } \\
\text { CS3 }\end{array}$ & $\begin{array}{l}\text { BH CS3 - FB } \\
\text { CS3 }\end{array}$ & $\begin{array}{l}\mathrm{BH} S 2-\mathrm{BH} \\
\mathrm{CS} 3\end{array}$ & $\begin{array}{l}\text { BH S2 - FB } \\
\text { CS3 }\end{array}$ & $\begin{array}{l}\mathrm{BH} \text { CS3 - FB } \\
\text { CS3 }\end{array}$ \\
\hline LV EDV (ml) & $127.2 \pm 46.2$ & $125.2 \pm 45.8$ & $130.2 \pm 45.9$ & $2.0 \pm 6.9$ & $-3.0 \pm 6.8$ & $-5.0 \pm 7.7$ & 0.142 & $0.036^{*}$ & $0.003^{*}$ \\
\hline $\begin{array}{l}\text { LV EDV / } \\
\text { BSA }\end{array}$ & $79.3 \pm 18.7$ & $77.8 \pm 17.5$ & $81.0 \pm 17.6$ & $1.5 \pm 4.7$ & $-1.8 \pm 4.2$ & $-3.3 \pm 5.1$ & 0.122 & $0.043^{*}$ & $0.003^{*}$ \\
\hline LV ESV (ml) & $52.0 \pm 21.7$ & $50.8 \pm 21.2$ & $54.0 \pm 22.6$ & $1.2 \pm 4.4$ & $-2.0 \pm 4.8$ & $-3.2 \pm 4.3$ & 0.191 & $0.042^{*}$ & $<0.001^{*}$ \\
\hline $\begin{array}{l}\text { LV ESV / } \\
\text { BSA }\end{array}$ & $32.2 \pm 9.4$ & $31.4 \pm 8.6$ & $33.4 \pm 9.3$ & $0.8 \pm 3.0$ & $-1.1 \pm 2.9$ & $-2.0 \pm 2.7$ & 0.184 & 0.058 & $0.001^{*}$ \\
\hline LV SV & $75.3 \pm 27.0$ & $74.4 \pm 27.4$ & $76.2 \pm 26.1$ & $0.9 \pm 5.8$ & $-0.9 \pm 6.4$ & $-1.8 \pm 6.0$ & 0.440 & 0.463 & 0.131 \\
\hline LV SV / BSA & $47.0 \pm 11.4$ & $46.4 \pm 11.2$ & $47.7 \pm 10.8$ & $0.7 \pm 3.6$ & $-0.6 \pm 4.2$ & $-1.3 \pm 3.9$ & 0.351 & 0.458 & 0.098 \\
\hline LV EF (\%) & $59.6 \pm 5.5$ & $59.7 \pm 6.1$ & $59.1 \pm 6.2$ & $-0.2 \pm 2.8$ & $0.5 \pm 3.5$ & $0.7 \pm 2.4$ & 0.748 & 0.480 & 0.172 \\
\hline LV Mass (g) & $63.2 \pm 20.3$ & $65.2 \pm 20.9$ & $63.6 \pm 21.0$ & $-2.0 \pm 4.3$ & $-0.4 \pm 7.3$ & $1.6 \pm 6.6$ & $0.026^{*}$ & 0.764 & 0.237 \\
\hline $\begin{array}{l}\text { LV Mass / } \\
\text { BSA }\end{array}$ & $39.4 \pm 7.2$ & $40.5 \pm 7.0$ & $39.6 \pm 7.8$ & $-1.1 \pm 2.9$ & $-0.2 \pm 4.6$ & $1.0 \pm 4.0$ & 0.063 & 0.854 & 0.237 \\
\hline RV EDV (ml) & $121.1 \pm 44.6$ & $117.9 \pm 43.5$ & $123.9 \pm 45.8$ & $3.2 \pm 6.2$ & $-2.8 \pm 9.6$ & $-6.0 \pm 8.5$ & $0.015^{*}$ & 0.153 & $0.001^{*}$ \\
\hline $\begin{array}{l}\text { RV EDV / } \\
\text { BSA }\end{array}$ & $75.6 \pm 19.8$ & $73.3 \pm 18.1$ & $77.2 \pm 19.8$ & $2.3 \pm 4.2$ & $-1.6 \pm 6.0$ & $-3.9 \pm 5.7$ & $0.009^{*}$ & 0.196 & $0.002^{*}$ \\
\hline RV ESV (ml) & $51.9 \pm 22.6$ & $48.7 \pm 20.4$ & $52.9 \pm 22.5$ & $3.2 \pm 5.5$ & $-1.0 \pm 5.9$ & $-4.2 \pm 6.8$ & $0.007^{*}$ & 0.384 & $0.004^{*}$ \\
\hline $\begin{array}{l}\text { RV ESV / } \\
\text { BSA }\end{array}$ & $32.1 \pm 11.2$ & $30.1 \pm 9.7$ & $32.8 \pm 11.3$ & $2.0 \pm 3.7$ & $-0.7 \pm 3.9$ & $-2.7 \pm 4.8$ & $0.010^{*}$ & 0.391 & $0.008^{*}$ \\
\hline RV SV & $69.3 \pm 24.2$ & $69.2 \pm 24.5$ & $71.0 \pm 25.0$ & $0.0 \pm 5.3$ & $-1.7 \pm 5.9$ & $-1.8 \pm 3.9$ & 0.985 & 0.144 & $0.030^{*}$ \\
\hline RV SV / BSA & $43.5 \pm 10.6$ & $43.2 \pm 9.5$ & $44.4 \pm 10.0$ & $0.3 \pm 3.1$ & $-0.9 \pm 3.5$ & $-1.2 \pm 2.5$ & 0.621 & 0.203 & $0.024^{*}$ \\
\hline RV EF (\%) & $58.1 \pm 5.9$ & $59.5 \pm 4.8$ & $58.2 \pm 5.9$ & $-1.4 \pm 3.5$ & $-0.1 \pm 2.9$ & $1.2 \pm 3.6$ & 0.054 & 0.820 & 0.094 \\
\hline
\end{tabular}

Unless otherwise indicated, data are means \pm standard deviations

$B H$ breath-hold, $B S A$ body surface area, CS3 C-SENSE acceleration factor of 3, EDV end-diastolic volume, EF ejection fraction, ESV end-systolic volume, FB freebreathing, $L V$ left ventricle, $R V$ right ventricle, S2 SENSE acceleration factor of 2, SV stroke volume The * represents $p<0.05$ 

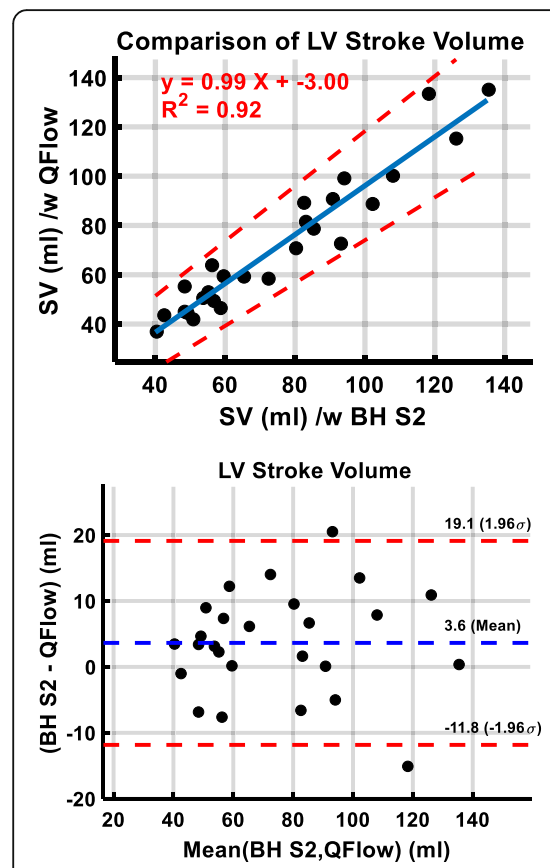

A
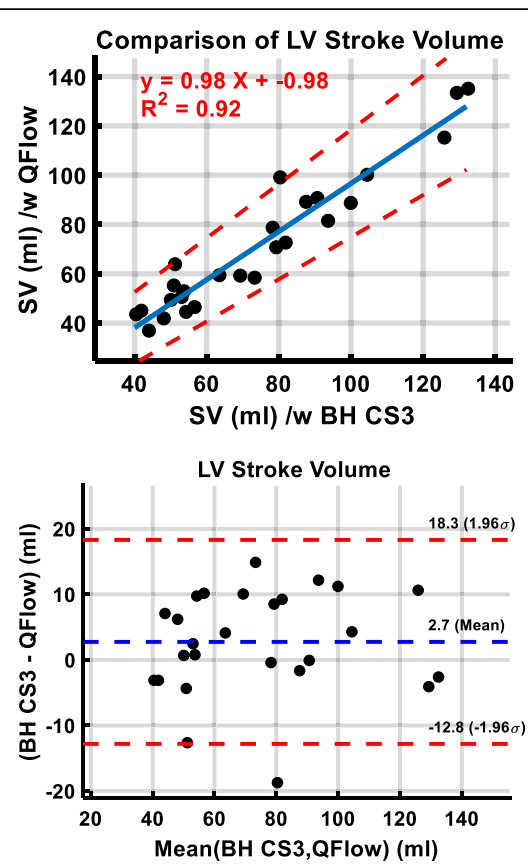

B
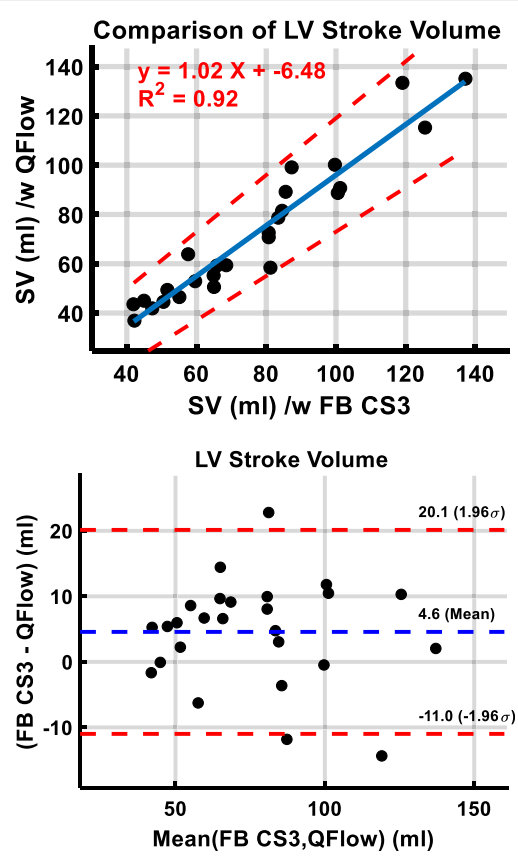

C

Fig. 2 Linear regression and Bland-Altman plots comparing left ventricular (LV) stroke volume (SV) measured with aortic quantitative flow with that measured from bSSFP cine short-axis images acquired with (a) BH SENSE $=2$, (b) BH C-SENSE $=3$, and BH breath-hold, CS3 C-SENSE acceleration factor of 3, FB free-breathing, S2 SENSE acceleration factor of 2

further analysis. Mean rank scores for BMC, EED, artifacts, and total image quality score with FB C-SENSE acquisitions were significantly lower $(p<0.005)$ than with the BH SENSE acquisitions (Fig. 1c). Mean rank scores for EED and combined total image quality scores with
BH C-SENSE were significantly lower $(p<0.05)$ than BH SENSE. Mean rank scores in each scoring criterion were comparable between BH SENSE and BH C-SENSE sequences. Figure 3 depicts the image quality scores for each scoring criteria. The combined image quality score

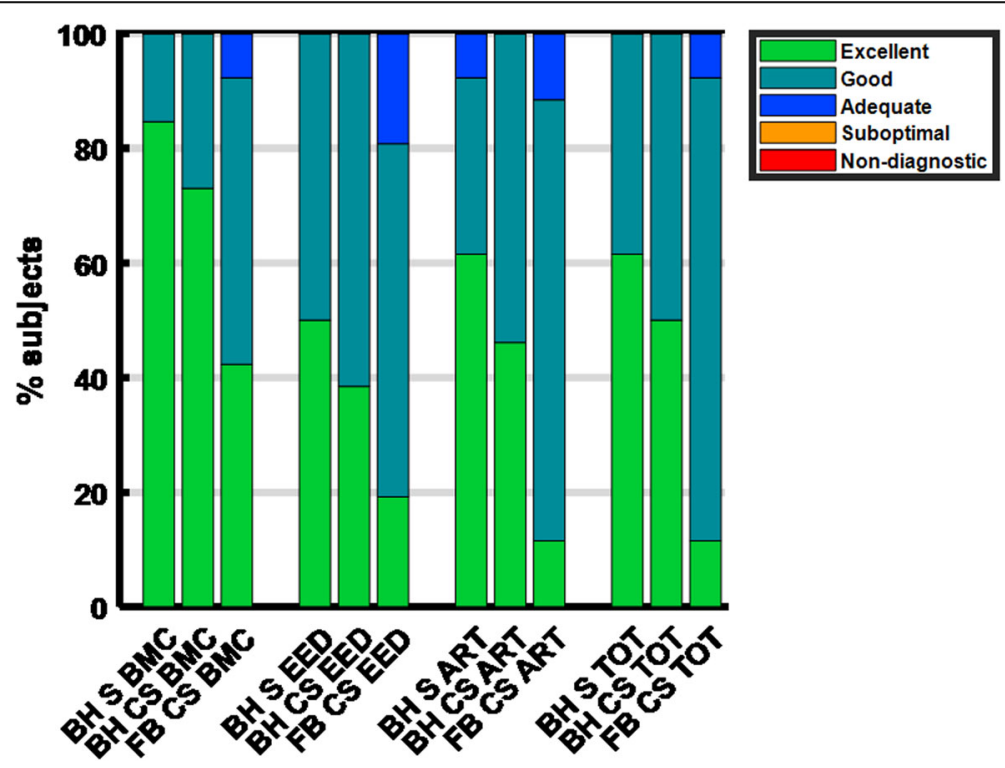

Fig. 3 Bar-plot analysis of image quality scores depicts percentage of patients who had image quality scores of excellent, good, adequate, suboptimal, or nondiagnostic in each grading criteria based on blood-to-myocardial contrast (BMC), endocardial edge definition (EED), and presence of artifacts (ART) and total (TOT) combined score. The combined image quality score is the equal-weight average of the three scores, which underscores the overall performance of the technique 
was excellent (16 of 26) to good (10 of 26) with $\mathrm{BH}$ SENSE, excellent (13 of 26) to good (13 of 26) with BH C-SENSE, and excellent (3 of 26) to good (21 of 26) to adequate (2 of 26) with FB C-SENSE (Fig. 3). For the combined image quality score, the difference between BH SENSE and BH C-SENSE was $(0.08 \pm 0.18$ (range: $0.50-0.39$ ) and the difference between BH SENSE and FB C-SENSE was $(0.42 \pm 0.34$ (range: -0.26 - 1.17). Figure 4 shows representative images with excellent, good, and adequate combined image quality scores using all three acquisition techniques.

\section{Discussion}

The results of this retrospective study demonstrated that image quality and biventricular volumetric indices using cine bSSFP acquisition with C-SENSE acceleration factor of 3 , either during $\mathrm{BH}$ or $\mathrm{FB}$, are comparable to the standard of care BH cine bSSFP acquisition with SENSE acceleration factor of 2. Both BH and FB cine bSSFP sequences with CSENSE $=3$ had spatio-temporal resolution nominally identical to the standard of care $\mathrm{BH}$ sequence and provided diagnostic image quality in all 26 patients encompassing a wide range of body sizes and heart rates. The $\mathrm{BH}$ duration was reduced by $33 \%$ using C-SENSE $=3$ compared to $\mathrm{SENSE}=2$. The total imaging time for the $\mathrm{FB}$ acquisition with C-SENSE $=3$ was comparable to SENSE $=2$.

Although the standard $\mathrm{BH}$ cine bSSFP sequences are well established, accelerated acquisition with shorter $\mathrm{BH}$ times or without $\mathrm{BHs}$ helps with imaging children and severely ill or sedated patients. Numerous strategies for undersampling $\mathrm{k}$-space in the time domain using either regular or irregular patterns in combination with either prospective cardiac gating or real-time cine imaging have been reported to provide diagnostic image quality and comparable ventricular volumetric assessment [20, 21, 23-26, 39-43]. However, studies with temporal undersampling schemes have reported underestimation of the LV mass and bias in both stroke volume and ejection fraction [23-26]. Some of the difference in volumetric indices can be attributed to experimental and

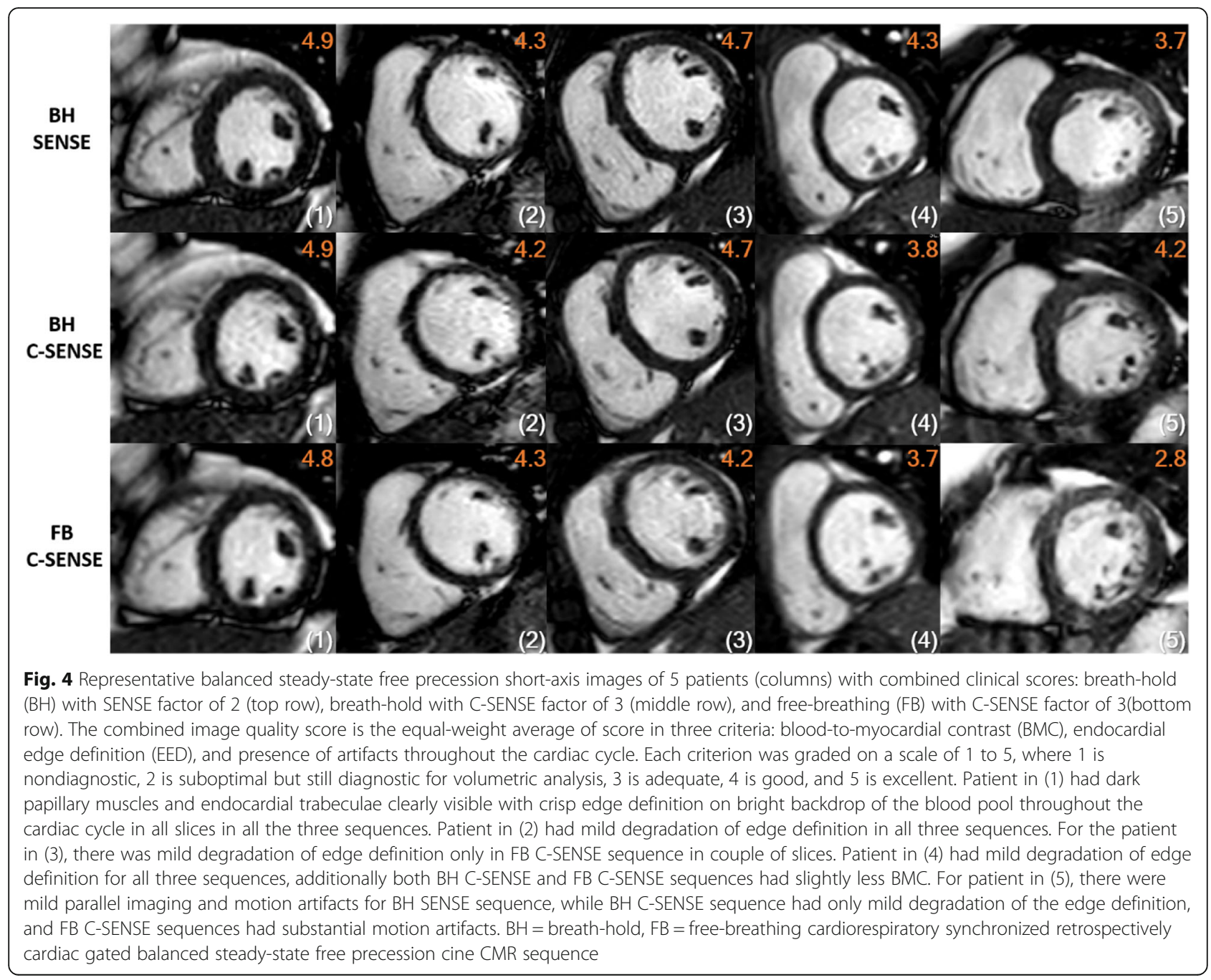


physiologic variations. However, bias in volumetric measurements is partly due to the decrease in effective temporal resolution when using temporal undersampling. Systematic evaluation of incremental increases in temporal undersampling using different reconstruction approaches on fully sampled cine bSSFP data has shown that these biases worsen with increasing acceleration factors and can be larger than physiologic variations [44]. In our study, bias and standard deviations for volumetric indices of both $\mathrm{BH}$ and $\mathrm{FB}$ C-SENSE $=3$ sequences compared to standard of care $\mathrm{BH}$ SENSE $=2$ sequence are comparable to the inter- and intra-observer values reported in the literature $[19,26,35,45-47]$. The overestimation of LV stroke volume by SAx measurements compared to aortic flow is 4 to $7 \%$ of the LV mass, which is comparable to reported LV trabeculation mass value of $8.2 \%$ seen in healthy subjects [48]. A consistent, small, non-zero velocity offset in aortic phase contrast data due to double-oblique slice orientation at the sinotubular junction may also have contributed to this difference. Overall, the statistically significant but small absolute difference seen in ventricular volumes between $\mathrm{BH}$ and $\mathrm{FB}$ is not clinically significant.

Although biventricular volumetric indices and LV mass were comparable across the three acquisitions, the endocardial delineation of both the BH and FB C-SENSE acquisitions were slightly inferior to BH SENSE. This suggests that spatial blurring caused by spatial domain pseudorandom sampling likely contributes to the differences in RV volumetric indices, exacerbated by the irregular RV contour shape. Decrease in blood-to-myocardial contrast can be attributed to diminished blood pool signal due to increased undersampling. The differences in BMC image quality between $\mathrm{BH}$ and $\mathrm{FB}$ C-SENSE suggest inferior attainment of steady state in FB sequence due to longitudinal through plane motion during the cardiac cycle, particularly in ventricular basal slices. The variability in depth of breathing may also contribute to the decrease in EED and artifact scores for FB compared to BH C-SENSE. This is noted frequently in our patients with muscular dystrophy and pectus excavatum. Another artifact noticed in larger patients was residual parallel lines next to bSSFP black bands at the edges of the field of view in images with C-SENSE factor of 3.

In addition to the noise penalty associated with the undersampling, the noise is further amplified in parallel imaging due to the nonorthogonality of the coil sensitivity profiles reflected by geometry or g-factors [49]. In 2D cartesian parallel imaging techniques, the image degradation due to noise increases exponentially around critical reduction factor of 3 to 4 [50]. A recent study in healthy adults using C-SENSE factor of 4 for cine bSSFP imaging showed adequate image quality; however, the acquired voxel size of $2.8 \mathrm{~mm}$ in phase encode direction was significantly larger than $1.6-1.7 \mathrm{~mm}$ used in our pediatric population [30]. Additionally, that study depended on shallow breathing while our study used explicit respiratory gating. In order to minimize the spatial blurring that occurs with iterative reconstruction from pseudo randomly sampled kspace data while trying to accelerate image acquisition, we conservatively tested a C-SENSE factor of 3 in this study. This study showed that the C-SENSE factor of 3 shortened the breath-hold time for SAx cine bSSFP acquisitions significantly while maintaining adequate LV and RV myocardial border definition. We showed good agreement of volumetric indices with those acquired with SENSE factor of 2 . This reduction in acquisition time can be traded for either faster patient throughput by acquiring additional slices per $\mathrm{BH}$ or to accommodate patients with impaired BH capacity.

Furthermore, the study demonstrated that C-SENSE acquisitions can be used for quantitative ventricular assessment along with FB cardiorespiratory synchronized cine bSSFP. Although study population consisted of children and young adults with structurally normal hearts, the sequence has potential utility for FB cardiorespiratory synchronized cine bSSFP in combination with $\mathrm{C}$ SENSE in older adults as well, especially patients with impaired BH capacity or capability. There is nothing about the sequence or acquisition scheme that would preclude large or older patients.

There are few limitations to this study. First, we did not acquire the cine bSSFP data with full $\mathrm{k}$-space sampling. Quantitative aortic flow was used as an internal reference to partially address this limitation. Second, the study population with structurally normal hearts mostly consisted of pectus and muscular dystrophy patients, both of which have male predominance. Third, the data acquisition for this retrospective study was performed as part of clinical scan sessions, so actual scan time comparisons between $\mathrm{BH}$ acquisitions is confounded by the irregular gaps between consecutive BHs. Lastly, SNR between different acquisitions was not compared quantitatively given the complex spatial distribution of noise in C-SENSE due to both data pseudorandom acquisition and iterative reconstruction. Differences in SNR were partially incorporated in the BMC measurements.

\section{Conclusion}

Cine bSSFP imaging with compressed SENSE acceleration factor of 3 reduces BH times by $33 \%$ compared to a clinically established SENSE acceleration factor of 2 with nominally identical spatio-temporal resolution while providing comparable LV and RV volumetric and functional indices and image quality. Also, C-SENSE combined with cardiorespiratory synchronized free-breathing cine bSSFP imaging provides comparable LV and RV volumetric and functional indices to that with clinically established $\mathrm{BH}$ 
acquisition with SENSE factor of 2 in comparable time. This reduction in acquisition time can be traded for either faster scan sessions with more slices per breath-hold or for reduction or elimination of BHs to accommodate patients with impaired BH capacity.

\section{Supplementary information}

Supplementary information accompanies this paper at https://doi.org/10. 1186/s12968-020-00642-y.

\section{Additional file 1 \\ Additional file 2. \\ Additional file 3 . \\ Additional file 4. \\ Additional file 5 . \\ Additional file 6 .}

\section{Abbreviations}

BH: Breath hold; BMC: Blood-to-myocardial contrast; BSA: Body surface area; bSSFP: Balanced steady state free precession; C-SENSE: Compressed sensitivity encoding; CMR: Cardiovascular magnetic resonance; EDV: Enddiastlic volume; EED: Endocardial edge definition; FA: Flip angle; FB: Free breathing; LV: Left ventricle/left ventricular; RV: Right ventricle/right ventricular; SAx: Short axis; SENSE: Sensitivity encoding; SNR: Signal-to-noise ratio; TE: Echo time; TR: Repetition time

\section{Acknowledgements}

Dr. Ryan Moore, Dr. Sean Lang, Dr. Justin Tretter, Dr. Eric Crotty, and Dr. Robert Fleck for their support in image acquisition.

\section{Authors' contributions}

MK: data collection, data analysis, and drafting the manuscript. AP: pulse sequence design and implementation, statistical analysis, and drafting the manuscript. HW: pulse sequence implementation and drafting the manuscript. TA: study design, data collection, data analysis, and drafting the manuscript. MDT: study design, data collection and drafting the manuscript MR: study design, data collection, data analysis, and drafting the manuscript. The author(s) read and approved the final manuscript.

\section{Funding}

Not applicable.

\section{Availability of data and materials}

The datasets generated and/or analyzed during the current study are not publicly available due to patient privacy concern and institutional policies but are available from the corresponding author on reasonable request.

\section{Ethics approval and consent to participate}

The Cincinnati Children's Hospital Medical Center Committee on Clinical Investigation approved this retrospective study and waived the requirement for informed consent.

\section{Consent for publication}

Not applicable.

\section{Competing interests}

HW: Employee of Philips Healthcare.

Author employed by the CMR scanner manufacturer was not part of the clinical data acquisition and/or image quality assessment.

\section{Author details}

'Department of Radiology, Cincinnati Children's Hospital Medical Center, 3333 Burnet Ave, Cincinnati, OH 45229, USA. ${ }^{2}$ Department of Radiology, University of Cincinnati College of Medicine, Cincinnati, OH, USA. ${ }^{3}$ MR Clinical Science, Philips Healthcare, Cincinnati, OH, USA. ${ }^{4}$ The Heart Institute, Cincinnati Children's Hospital Medical Center, Cincinnati, OH, USA
${ }^{5}$ Department of Pediatrics, University of Cincinnati College of Medicine, Cincinnati, OH, USA.

Received: 4 March 2020 Accepted: 29 May 2020

Published online: 27 July 2020

References

1. Dodge HT, Baxley WA. Left ventricular volume and mass and their significance in heart disease. Am J Cardiol. 1969;23(4):528-37. https://doi. org/10.1016/0002-9149(69)90006-x.

2. White HD, Norris RM, Brown MA, Brandt PW, Whitlock RM, Wild CJ. Left ventricular end-systolic volume as the major determinant of survival after recovery from myocardial infarction. Circulation. 1987;76(1):44-51. https:// doi.org/10.1161/01.cir.76.1.44

3. Taniguchi K, Nakano S, Hirose H, Matsuda H, Shirakura R, Sakai K, Kawamoto T, Sakaki S, Kawashima Y. Preoperative left ventricular function: Minimal requirement for successful late results of valve replacement for aortic regurgitation. J Am Coll Cardiol. 1987;10(3):510-8. https://doi.org/10.1016/ S0735-1097(87)80192-4.

4. Levy D, Garrison RJ, Savage DD, Kannel WB, Castelli WP. Prognostic implications of echocardiographically determined left ventricular mass in the Framingham heart study. N Engl J Med. 1990;322(22):1561-6. https:// doi.org/10.1056/nejm199005313222203

5. Solomon SD, Anavekar N, Skali H, McMurray JJ, Swedberg K, Yusuf S, Granger CB, Michelson EL, Wang D, Pocock S, Pfeffer MA. Influence of ejection fraction on cardiovascular outcomes in a broad spectrum of heart failure patients. Circulation. 2005;112(24):3738-44. https://doi.org/10.1161/ circulationaha.105.561423.

6. Valsangiacomo Buechel ER, Grosse-Wortmann L, Fratz S, Eichhorn J, Sarikouch S, Greil GF, Beerbaum P, Bucciarelli-Ducci C, Bonello B, Sieverding L, Schwitter J, Helbing WA, Galderisi M, Miller O, Sicari R, Rosa J, Thaulow E, Edvardsen T, Brockmeier K, Qureshi S, Stein J. Indications for cardiovascular magnetic resonance in children with congenital and acquired heart disease: an expert consensus paper of the Imaging Working Group of the AEPC and the Cardiovascular Magnetic Resonance Section of the EACVI. Eur Heart J Cardiovasc Imaging. 2015;16(3):281-97. https://doi.org/10.1093/ehjci/jeu129.

7. Bellenger NG, Burgess MI, Ray SG, Lahiri A, Coats AJ, Cleland JG, Pennell DJ. Comparison of left ventricular ejection fraction and volumes in heart failure by echocardiography, radionuclide ventriculography and cardiovascular magnetic resonance; are they interchangeable? Eur Heart J. 2000;21(16): 1387-96.

8. Mannaerts HF, Van Der Heide JA, Kamp O, Papavassiliu T, Marcus JT, Beek A Van Rossum AC, Twisk J, Visser CA. Quantification of left ventricular volumes and ejection fraction using freehand transthoracic three-dimensional echocardiography: comparison with magnetic resonance imaging. J Am Soc Echocardiogr. 2003;16(2):101-9. https://doi.org/10.1067/mje.2003.7.

9. van den Bosch AE, Robbers-Visser D, Krenning BJ, McGhie JS, Helbing WA, Meijboom FJ, Roos-Hesselink JW. Comparison of real-time threedimensional echocardiography to magnetic resonance imaging for assessment of left ventricular mass. Am J Cardiol. 2006;97(1):113-7. https:// doi.org/10.1016/j.amjcard.2005.07.114.

10. Puntmann VO, Gebker R, Duckett S, Mirelis J, Schnackenburg B, Graefe M, Razavi R, Fleck E, Nagel E. Left ventricular chamber dimensions and wall thickness by cardiovascular magnetic resonance: comparison with transthoracic echocardiography. Eur Heart J Cardiovasc Imaging. 2013;14(3): 240-6. https://doi.org/10.1093/ehjci/jes145.

11. Delgado JA, Abad P, Rascovsky S, Calvo V, Castrillon G, Greil G, Uribe S. Assessment of cardiac volumes using an isotropic whole-heart dual cardiac phase sequence in pediatric patients. J Magn Reson Imaging. 2014;39(3): 708-16. https://doi.org/10.1002/jmri.24203.

12. Barkhausen J, Ruehm SG, Goyen M, Buck T, Laub G, Debatin JF. MR evaluation of ventricular function: true fast imaging with steady-state precession versus fast low-angle shot cine MR imaging: feasibility study. Radiology. 2001;219(1): 264-9. https:/doi.org/10.1148/radiology.219.1.r01ap12264.

13. Plein S, Bloomer TN, Ridgway JP, Jones TR, Bainbridge GJ, Sivananthan MU. Steady-state free precession magnetic resonance imaging of the heart: comparison with segmented k-space gradient-echo imaging. J Magn Reson Imaging. 2001;14(3):230-6. https://doi.org/10.1002/jmri.1178.

14. Scheffler K, Lehnhardt S. Principles and applications of balanced SSFP techniques. Eur Radiol. 2003;13(11):2409-18. https://doi.org/10.1007/s00330003-1957-x. 
15. van den Brink JS, Watanabe Y, Kuhl CK, Chung T, Muthupillai R, Van Cauteren M, Yamada K, Dymarkowski S, Bogaert J, Maki JH, Matos C Casselman JW, Hoogeveen RM. Implications of SENSE MR in routine clinical practice. Eur J Radiol. 2003;46(1):3-27. https://doi.org/10.1016/s0720048x(02)00333-9.

16. Kacere RD, Pereyra M, Nemeth MA, Muthupillai R, Flamm SD. Quantitative assessment of left ventricular function: steady-state free precession MR imaging with or without sensitivity encoding. Radiology. 2005;235(3):10315. https://doi.org/10.1148/radiol.2353030995.

17. Geva T. Is MRI the preferred method for evaluating right ventricular size and function in patients with congenital heart disease?: MRI is the preferred method for evaluating right ventricular size and function in patients with congenital heart disease. Circ Cardiovasc Imaging. 2014;7(1):190-7. https:// doi.org/10.1161/CIRCIMAGING.113.000553.

18. Grothues F, Moon JC, Bellenger NG, Smith GS, Klein HU, Pennell DJ. Interstudy reproducibility of right ventricular volumes, function, and mass with cardiovascular magnetic resonance. Am Heart J. 2004;147(2):218-23. https://doi.org/10.1016/j.ahj.2003.10.005.

19. Gandy SJ, Waugh SA, Nicholas RS, Simpson HJ, Milne W, Houston JG. Comparison of the reproducibility of quantitative cardiac left ventricular assessments in healthy volunteers using different MRI scanners: a multicenter simulation. J Magn Reson Imaging. 2008;28(2):359-65. https:// doi.org/10.1002/jmri.21401.

20. Jahnke C, Nagel E, Gebker R, Bornstedt A, Schnackenburg B, Kozerke S, Fleck E, Paetsch I. Four-dimensional single breathhold magnetic resonance imaging using kt-BLAST enables reliable assessment of left- and rightventricular volumes and mass. J Magn Reson Imaging. 2007;25(4):737-42. https://doi.org/10.1002/jmri.20877.

21. Eberle HC, Nassenstein K, Jensen CJ, Schlosser T, Sabin GV, Naber CK, Bruder O. Rapid MR assessment of left ventricular systolic function after acute myocardial infarction using single breath-hold cine imaging with the temporal parallel acquisition technique (TPAT) and 4D guide-point modelling analysis of left ventricular function. Eur Radiol. 2010;20(1):7380. https://doi.org/10.1007/s00330-009-1522-3.

22. Young AA, Cowan BR, Schoenberg SO, Wintersperger BJ. Feasibility of single breath-hold left ventricular function with 3 tesla TSENSE acquisition and 3D modeling analysis. J Cardiovasc Magn Reson. 2008;10(1):24. https://doi.org/ 10.1186/1532-429X-10-24.

23. Goebel J, Nensa F, Schemuth HP, Maderwald S, Gratz M, Quick HH, Schlosser T, Nassenstein K. Compressed sensing cine imaging with high spatial or high temporal resolution for analysis of left ventricular function. J Magn Reson Imaging. 2016;44(2):366-74. https://doi.org/10. 1002/jmri.25162.

24. Vincenti G, Monney P, Chaptinel J, Rutz T, Coppo S, Zenge MO, Schmidt M, Nadar MS, Piccini D, Chevre P, Stuber M, Schwitter J. Compressed sensing single-breath-hold CMR for fast quantification of LV function, volumes, and mass. JACC Cardiovasc Imaging. 2014;7(9):882-92. https://doi.org/10.1016/j. jcmg.2014.04.016.

25. Kido T, Kido T, Nakamura M, Watanabe K, Schmidt M, Forman C, Mochizuki T. Assessment of left ventricular function and mass on Free-breathing compressed sensing real-time cine imaging. Circ J. 2017;81(10):1463-8. https://doi.org/10.1253/circj.CJ-17-0123.

26. Lin ACW, Strugnell W, Riley R, Schmitt B, Zenge M, Schmidt M, Morris NR, Hamilton-Craig C. Higher resolution cine imaging with compressed sensing for accelerated clinical left ventricular evaluation. J Magn Reson Imaging. 2017:45(6):1693-9. https://doi.org/10.1002/jmri.25525.

27. Pednekar AS, Wang H, Flamm S, Cheong BY, Muthupillai R. Two-center clinical validation and quantitative assessment of respiratory triggered retrospectively cardiac gated balanced-SSFP cine cardiovascular magnetic resonance imaging in adults. J Cardiovasc Magn Reson. 2018; 20(1):44. https://doi.org/10.1186/s12968-018-0467-6.

28. Pednekar AJS, Noel C, Masand P. Free-breathing Cardiorespiratory Synchronized Cine MRI for Assessment of Left and Right Ventricular Volume and Function in Sedated Children and Adolescents with Impaired Breath-holding Capacity. Radiol: Cardiothorac Imaging. 2019; 1(2):e180027. https://doi.org/10.1148/ryct.2019180027.

29. Sartoretti E, Sartoretti T, Binkert C, Najafi A, Schwenk A, Hinnen M, van Smoorenburg L, Eichenberger B, Sartoretti-Schefer S. Reduction of procedure times in routine clinical practice with compressed SENSE magnetic resonance imaging technique. PLoS One. 2019;14(4):e0214887. https://doi.org/10.1371/journal.pone.0214887.
30. Ma Y, Hou Y, Ma Q, Wang X, Sui S, Wang B. Compressed SENSE singlebreath-hold and free-breathing cine imaging for accelerated clinical evaluation of the left ventricle. Clin Radiol. 2019;74(4):325.e329-17. https:// doi.org/10.1016/j.crad.2018.12.012.

31. Geerts-Ossevoort $L$, de Weerdt E, Duijndam A, van ljperen G, Peeters $H$, Doneva M, Nijenhuis M, Huang A. Compressed SENSE. Speed done right. Every time. Philips Field Strength Magazine. 2018. p. 6619. https:// philipsproductcontent.blob.core.windows.net/assets/20180109/619119731f2 a42c4acd4a863008a46c7.pdf.

32. Bieri O, Markl M, Scheffler K. Analysis and compensation of eddy currents in balanced SSFP. Magn Reson Med. 2005;54(1):129-37. https://doi.org/10. 1002/mrm.20527.

33. Lorenz CH, Walker ES, Morgan VL, Klein SS, Graham TP Jr. Normal human right and left ventricular mass, systolic function, and gender differences by cine magnetic resonance imaging. J Cardiovasc Magn Reson. 1999;1(1):7-21. https://doi.org/10.3109/10976649909080829.

34. Alfakih K, Plein S, Thiele H, Jones T, Ridgway JP, Sivananthan MU. Normal human left and right ventricular dimensions for MRI as assessed by turbo gradient echo and steady-state free precession imaging sequences. J Magn Reson Imaging. 2003;17(3):323-9.

35. Hudsmith LE, Petersen SE, Francis JM, Robson MD, Neubauer S. Normal human left and right ventricular and left atrial dimensions using steady state free precession magnetic resonance imaging. J Cardiovasc Magn Reson. 2005;7(5):775-82. https://doi.org/10.1080/10976640500295516.

36. Miller S, Simonetti OP, Carr J, Kramer U, Finn JP. MR imaging of the heart with cine true fast imaging with steady-state precession: influence of spatial and temporal resolutions on left ventricular functional parameters. Radiology. 2002;223(1):263-9. https://doi.org/10.1148/radiol.2231010235.

37. Bland JM, Altman DG. Statistical methods for assessing agreement between two methods of clinical measurement. Lancet. 1986;1(8476):307-10.

38. McGill R, Tukey JW, Larsen WA. Variations of box plots. Am Stat. 1978;32(1): 12-6. https://doi.org/10.2307/2683468.

39. Sudarski S, Henzler T, Haubenreisser H, Dosch C, Zenge MO, Schmidt M, Nadar MS, Borggrefe M, Schoenberg SO, Papavassiliu T. Free-breathing sparse sampling cine MR imaging with iterative reconstruction for the assessment of left ventricular function and mass at 3.0 T. Radiology. 2017; 282(1):74-83. https://doi.org/10.1148/radiol.2016151002.

40. Feng L, Srichai MB, Lim RP, Harrison A, King W, Adluru G, Dibella EV, Sodickson DK, Otazo R, Kim D. Highly accelerated real-time cardiac cine MRI using k-t SPARSE-SENSE. Magn Reson Med. 2013;70(1):64-74. https://doi.org/ 10.1002/mrm.24440

41. Aandal G, Nadig V, Yeh V, Rajiah P, Jenkins T, Sattar A, Griswold M, Gulani V, Gilkeson RC, Seiberlich N. Evaluation of left ventricular ejection fraction using through-time radial GRAPPA. J Cardiovasc Magn Reson. 2014;16(1):79. https://doi.org/10.1186/s12968-014-0079-8.

42. Muthurangu V, Lurz P, Critchely JD, Deanfield JE, Taylor AM, Hansen MS. Real-time assessment of right and left ventricular volumes and function in patients with congenital heart disease by using high spatiotemporal resolution radial k-t SENSE. Radiology. 2008;248(3):782-91. https://doi.org/10. 1148/radiol.2482071717.

43. Steeden JA, Kowalik GT, Tann O, Hughes M, Mortensen KH, Muthurangu V. Real-time assessment of right and left ventricular volumes and function in children using high spatiotemporal resolution spiral bSSFP with compressed sensing. J Cardiovasc Magn Reson. 2018;20(1):79. https://doi.org/10.1186/ s12968-018-0500-9.

44. Yoon J-H, Kim P-K, Yang Y-J, Park J, Choi BW, Ahn C-B. Biases in the assessment of left ventricular function by compressed sensing cardiovascular cine MRI. Investig Magn Reson Imaging. 2019;23(2):11424.

45. Karamitsos TD, Hudsmith LE, Selvanayagam JB, Neubauer S, Francis JM. Operator induced variability in left ventricular measurements with cardiovascular magnetic resonance is improved after training. J Cardiovasc Magn Reson. 2007;9(5):777-83. https://doi.org/10.1080/ 10976640701545073.

46. Pednekar AS, Muthupillai R, Cheong B, Flamm SD. Automatic computation of left ventricular ejection fraction from spatiotemporal information in cineSSFP cardiac MR images. J Magn Reson Imaging. 2008;28(1):39-50. https:// doi.org/10.1002/jmri.21363.

47. Sardanelli F, Quarenghi M, Di Leo G, Boccaccini L, Schiavi A. Segmentation of cardiac cine MR images of left and right ventricles: interactive semiautomated methods and manual contouring by two readers with 
different education and experience. J Magn Reson Imaging. 2008;27(4):78592. https://doi.org/10.1002/jmri.21292.

48. Captur G, Muthurangu V, Cook C, Flett AS, Wilson R, Barison A, Sado DM, Anderson S, McKenna WJ, Mohun TJ, Elliott PM, Moon JC. Quantification of left ventricular trabeculae using fractal analysis. J Cardiovasc Magn Reson. 2013;15(1):36. https://doi.org/10.1186/1532-429X-15-36.

49. Pruessmann KP, Weiger M, Scheidegger MB, Boesiger P. SENSE: sensitivity encoding for fast MRI. Magn Reson Med. 1999;42(5):952-62.

50. Kozerke S, Plein S. Accelerated CMR using zonal, parallel and prior knowledge driven imaging methods. J Cardiovasc Magn Reson. 2008;10(1): 29. https://doi.org/10.1186/1532-429X-10-29.

\section{Publisher's Note}

Springer Nature remains neutral with regard to jurisdictional claims in published maps and institutional affiliations.

Ready to submit your research? Choose BMC and benefit from:

- fast, convenient online submission

- thorough peer review by experienced researchers in your field

- rapid publication on acceptance

- support for research data, including large and complex data types

- gold Open Access which fosters wider collaboration and increased citations

- maximum visibility for your research: over $100 \mathrm{M}$ website views per year

At BMC, research is always in progress.

Learn more biomedcentral.com/submissions 